\title{
Endothelial cell-glucocorticoid receptor interactions and regulation of Wnt signaling
}

\author{
Han Zhou, ${ }^{1,2}$ Sameet Mehta, ${ }^{3}$ Swayam Prakash Srivastava, ${ }^{1,2}$ Kariona Grabinska, ${ }^{2,4}$ Xinbo Zhang, ${ }^{2,5,6}$ \\ Chris Wong, ${ }^{1}$ Ahmad Hedayat, ${ }^{1,2}$ Paola Perrotta, ${ }^{2,4}$ Carlos Fernández-Hernando, ${ }^{2,5,6,7}$ \\ William C. Sessa, ${ }^{2,4}$ and Julie E. Goodwin ${ }^{1,2}$ \\ ${ }^{1}$ Department of Pediatrics, ${ }^{2}$ Vascular Biology and Therapeutics Program, ${ }^{3}$ Department of Cenetics, ${ }^{4}$ Department of \\ Pharmacology, ${ }^{5}$ ntegrative Cell Signaling and Neurobiology of Metabolism Program, ${ }^{6}$ Department of Comparative \\ Medicine, and 'Department of Pathology, Yale University School of Medicine, New Haven, Connecticut, USA.
}

\begin{abstract}
Vascular inflammation is present in many cardiovascular diseases, and exogenous glucocorticoids have traditionally been used as a therapy to suppress inflammation. However, recent data have shown that endogenous glucocorticoids, acting through the endothelial glucocorticoid receptor, act as negative regulators of inflammation. Here, we performed ChIP for the glucocorticoid receptor, followed by next-generation sequencing in mouse endothelial cells to investigate how the endothelial glucocorticoid receptor regulates vascular inflammation. We identified a role of the Wnt signaling pathway in this setting and show that loss of the endothelial glucocorticoid receptor results in upregulation of Wnt signaling both in vitro and in vivo using our validated mouse model. Furthermore, we demonstrate glucocorticoid receptor regulation of a key gene in the Wnt pathway, $F r z b$, via a glucocorticoid response element gleaned from our genomic data. These results suggest a role for endothelial Wnt signaling modulation in states of vascular inflammation.
\end{abstract}

Conflict of interest: The authors have declared that no conflict of interest exists.

Copyright: ( 2020 , American Society for Clinical Investigation.

Submitted: June 27, 2019

Accepted: January 2, 2020

Published: February 13, 2020

Reference information: /CI Insight. 2020;5(3):e131384.

https://doi.org/10.1172/jci.

insight.131384.

\section{Introduction}

Inflammation is a complex cascade of adaptive cellular responses to injurious stimuli, which occurs in many cardiovascular diseases (1). Vascular inflammation is manifested in several ways, including enhanced expression of endothelial cell (EC) adhesion molecules, inflammatory cell recruitment, cytokine release, and impaired nitric oxide (NO) bioactivity $(2,3)$. Physiologically, endogenous glucocorticoids (GC, corticosterone in rodents and cortisol in humans) exert a permissive role in suppressing local and systemic inflammation. Administration of exogenous GC, such as hydrocortisone and dexamethasone (DEX), are widely used to suppress inflammation, although the mechanisms through which they act are not entirely clear (4). Administration of exogenous GCs as antiinflammatory agents provides systemic ligand to all cells expressing the glucocorticoid receptor (GR). Under these conditions, the particular role of a tissue-specific GR in resolving inflammation cannot be examined nor can the role of the endogenous ligand, cortisol. Additionally, side effects from systemic GC are common and can be severe, to the point of rendering them intolerable and therefore ineffective for vascular inflammatory disorders (5).

Previously, we have shown that endothelial GR is a negative regulator of vascular inflammation in models of sepsis (6) and atherosclerosis (7). In the latter case, mice lacking endothelial GR bred onto an ApoE-KO background developed more severe atherosclerotic lesions when fed a high-fat diet (HFD) compared with controls (7). Of note, the increase in lesion size and complexity could not be explained by changes in circulating lipids and is remarkable because it suggests that circulating cortisol via binding to endothelial GR is vasculoprotective. Mechanistically, we elucidate a unique GR DNA landscape by performing GR-specific ChIP followed by next-generation sequencing in primary ECs that directly associates GR activation with antiinflammatory pathways in the vascular endothelium. Importantly, endothelial GR represses a number of genes critical for the regulation of Wnt signaling pathway. This pathway is independent from that of NF- $\mathrm{kB}$, a classic target for GR, $(8,9)$ and therefore highlights the permissive effects of cortisol in physiologically relevant states. 


\section{Results}

Genome-wide GR DNA binding in ECs. To begin to understand the mechanisms by which GR regulates EC functions, we performed ChIP sequencing (ChIP-seq) for GR in primary mouse lung ECs (MLECs) using a commercially available, ChIP-quality GR antibody. Six conditions were submitted for GR ChIP-seq analysis as follows: (a) control siRNA-treated cells, (b) GR siRNA-treated cells, (c) control siRNA treated with DEX 100 nM for 1 hour, (d) GR siRNA treated with DEX 100 nM for 1 hour, (e) IgG control, and (f) whole cell input. The duration of DEX treatment was replicated from a previous ChIP study (10), and knockdown of GR using this siRNA is greater than $80 \%$ (6). The control siRNA DEX-treated sample was treated as the "control" sample, reflecting agonist activation of GR. Control siRNA samples were 2.63\% enriched in GR elements, which is within the expected range of $1 \%-7 \%$ for ChIP experiments (11); after treatment with GR siRNA, enrichment was reduced to $0.02 \%$, confirming excellent knockdown via siRNA and high specificity of the GR ChIP antibody.

Normalization of peaks to each $1,000,000 \mathrm{bp}$ reads resulted in the distribution shown in Figure $1 \mathrm{~A}$. There were approximately 35,000 GR-enriched peaks overall. As expected, the "control" sample in which GR was activated by DEX shows the greatest number of GR-enriched peaks, approximately 7-fold higher than the other conditions. The residual smaller peak present in the GR siRNA DEX-treated cells is approximately 5-fold less than the DEX GR replete conditions but approximately 2-fold higher than the other conditions. This was anticipated since this GR siRNA knockdown is not 100\% (6). The other 4 conditions tested are completely overlapping and represent background.

Analysis of the top 10,000 DEX-stimulated, GR-enriched peaks shows very tight localization around the nearest transcriptional start site (TSS, Figure 1B). Further refinement of the subset of peaks within 1000 $\mathrm{bp}$ of TSS resulted in the identification of 3 clusters of genes. Cluster 2, which was the largest group, had no discernible pattern of binding over the interval examined. Cluster 1 showed enriched binding slightly upstream of the TSS, and cluster 3 showed enriched binding at TSS (Figure 1C).

Peaks were also analyzed to determine the breakdown of the GR binding location based on genomic region. As shown in Figure 1D, the vast majority (87\%) of GR-enriched peaks are in introns, with about 7\% binding to promoter-TSS or TSS regions. Further analysis revealed that $97 \%$ of intronic GR-enriched peaks are found in protein-coding genes, while $84 \%$ of TSS GR sites map to protein coding genes. If intronic GR peaks are restricted to those within $5 \mathrm{~kb}$ of the TSS, there are 59 genes with GR binding upstream of the TSS and 361 genes with GR binding downstream of the TSS.

The analysis was further refined to examine the top 1000 peaks in more detail. First, peak binding sites were characterized by location, as shown in Figure 1E. Using motif detection algorithms, these 1000 peaks were queried for any plausible motifs by inputting the classic 6-bp palindromic GRE (5' - AGAACAnnnTGTTCT - 3', where n can be any base) (12) as a seed sequence, as well as searched for de novo motifs. Ninety genes (Supplemental Table 1; supplemental material available online with this article; https://doi. org/10.1172/jci.insight.131384DS1) resulted when searching by the classic GRE, and 81 genes resulted that contained a de novo motif (Supplemental Table 2). Cross-referencing both lists resulted in 65 genes (Supplemental Table 3) that possessed both motifs (Figure 1F). We suspected that peaks having both motifs may be enriched in certain regulatory elements or have other common features. When the peak binding location for these 65 genes was mapped, the vast majority were either intergenic or intronic (Figure 1G). Using the ENCODE ChIP-seq Significance Tool and the mm10 reference genome, the top 10 enriched ENCODE transcription factors within $\pm 500 \mathrm{bp}$ of the TSS $/ 5^{\prime}$ end were identified for this list of 65 genes (Supplemental Table 4). Interestingly, these genes were noted to have ChIP peak binding sites ranging from $-272 \mathrm{~kb}$ to $+400 \mathrm{~kb}$ from the TSS.

To assess if genes with ChIP peak binding sites closer to TSS had a similar TF profile, the top 1000 peaks for those binding within $\pm 1 \mathrm{~kb}$ from the TSS were also interrogated. This resulted in 29 genes (Supplemental Table 5) with the peak binding locations shown in Figure 2A. Each of these peaks and the surrounding $\pm 5 \mathrm{~kb}$ region were examined in detail in the ENCODE database. As shown in Figure 2B, transcription factor (TF) binding sites were abundant in these genes, regardless of whether the ChIP-seq binding occurred in the promoter-TSS or in another region. Notably, the 7 most abundant TFs detected have all been shown to interact with nuclear receptors (13-18), except for max1, though it is a binding partner of myc (19). Additionally, Myc, BLHLE40, max, and TCF12 are members of the basic-helix-loophelix (bHLH) family of nuclear receptor coactivators (20). Interestingly, when these genes were categorized according to how many regulatory elements (TF binding sites, enhancers, open chromatin), they had 

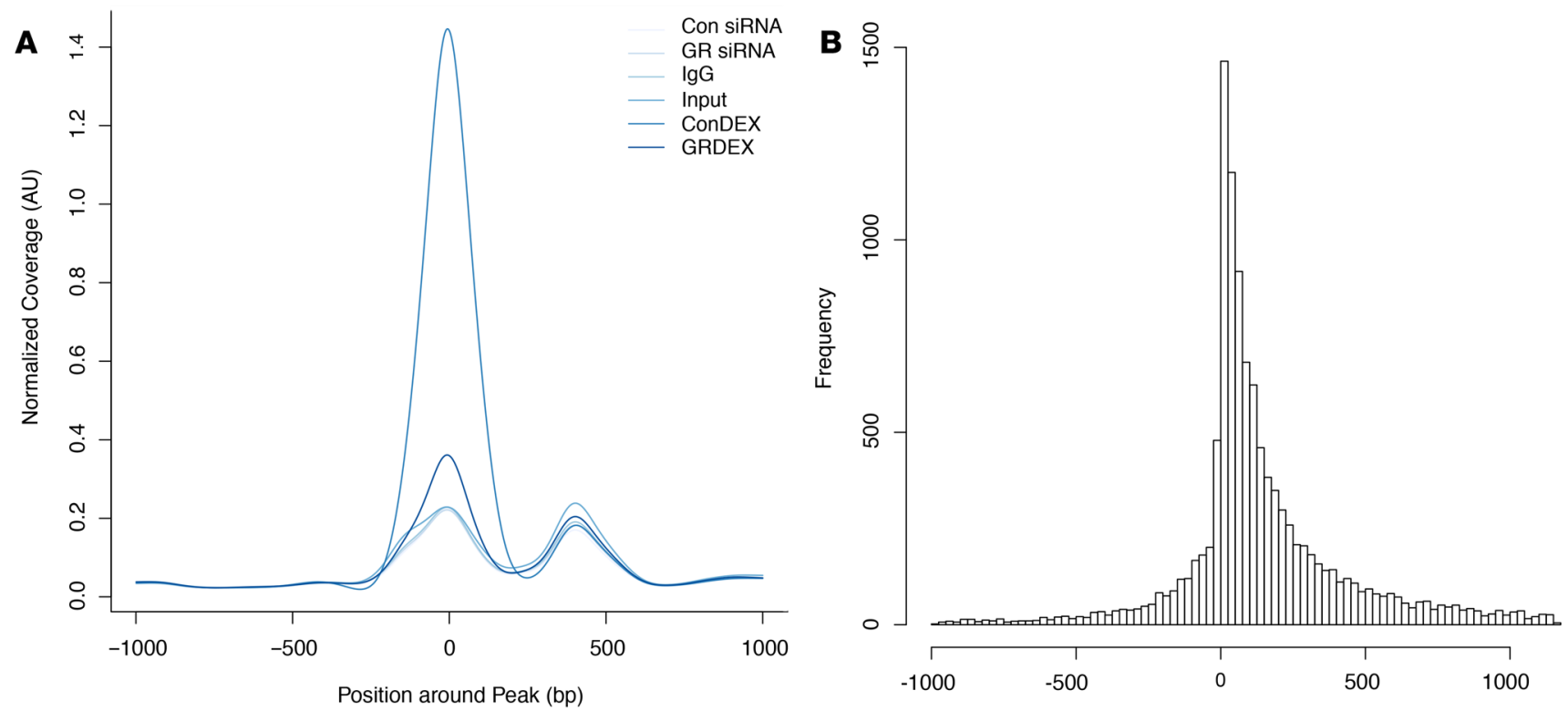

C

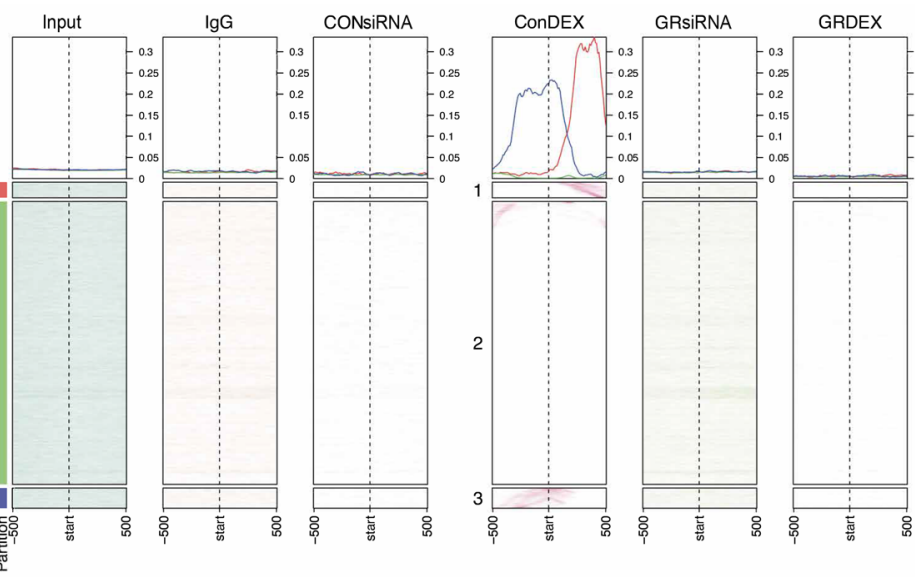

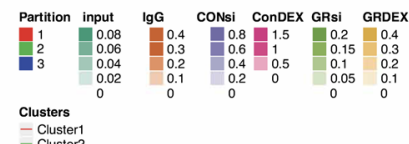
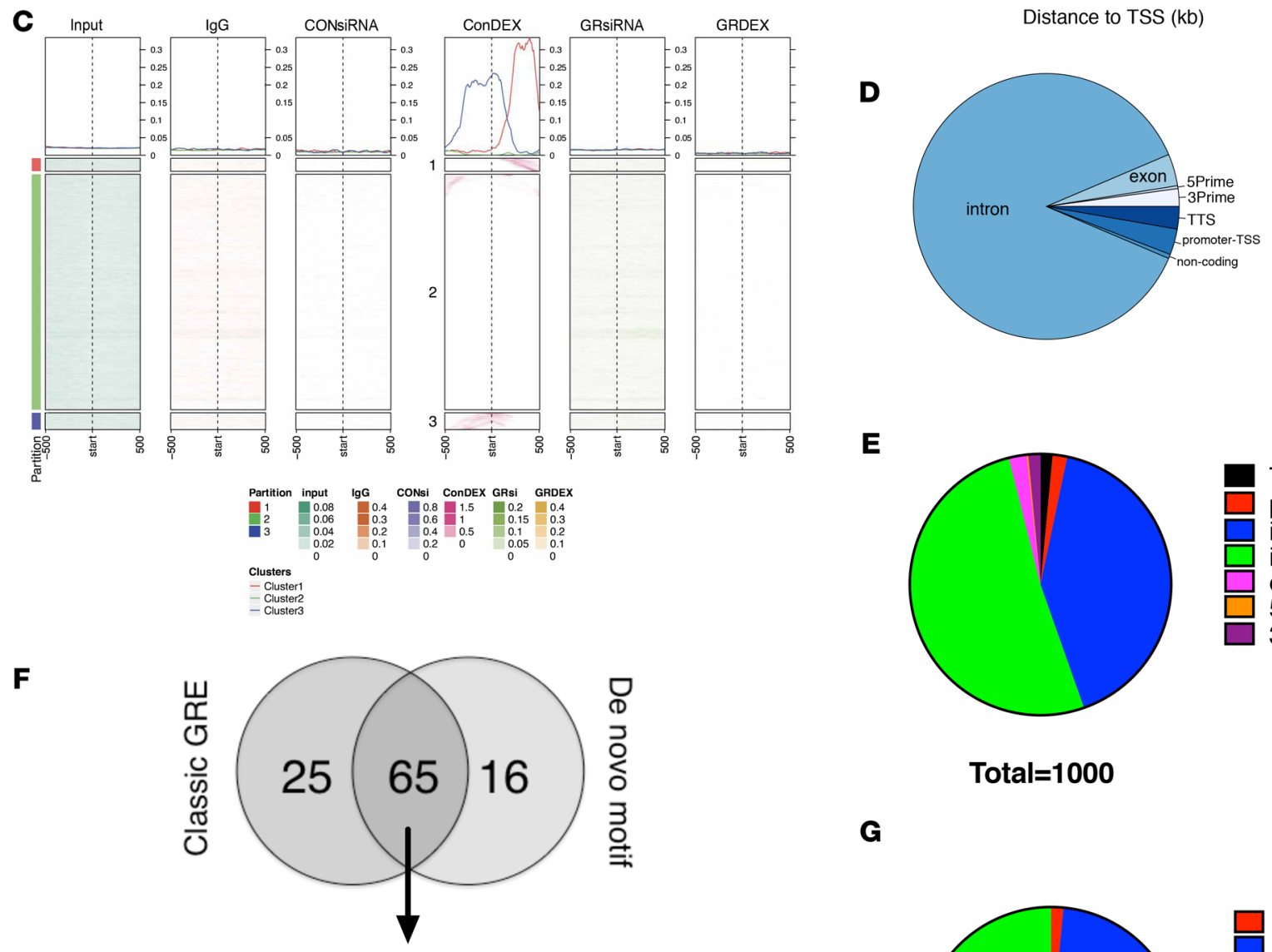

E

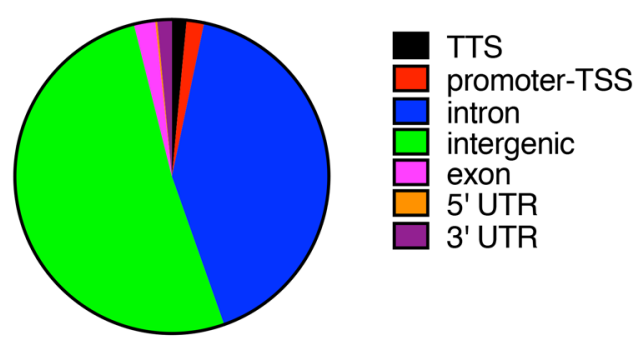

Total $=1000$

$\mathbf{G}$

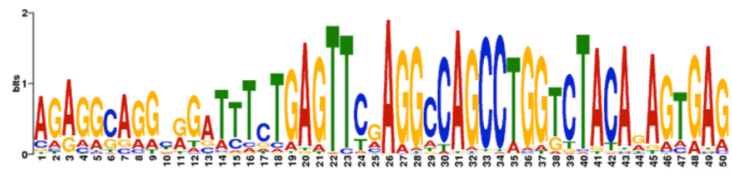

\section{and}
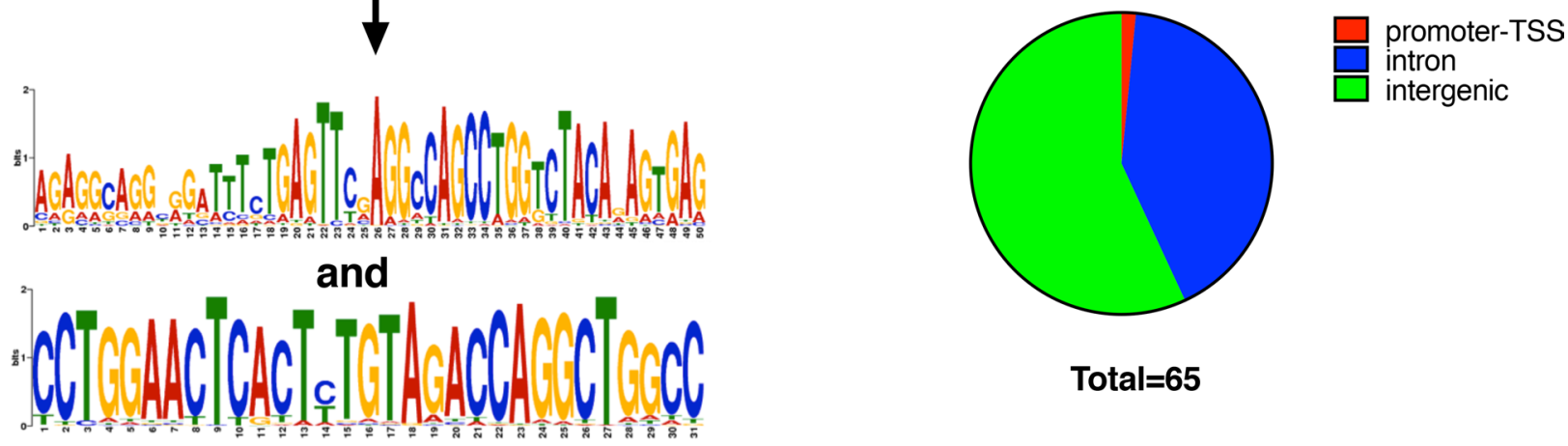

Total $=65$ 
Figure 1. ChIP-seq results. (A) Six conditions were submitted for ChIP-seq analysis. Control siRNA cells treated with dexamethasone (DEX) (ConDEX) showed the largest peak, reflecting binding of the glucocorticoid receptor (GR) by its ligand. A smaller peak was observed from the GR siRNA cells treated with DEX (GRDEX), as siRNA knockdown is not $100 \%$ complete. The other 4 conditions had overlapping peak profiles indicating nonspecific background. Normalized coverage per 1,000,000 reads is plotted as a function of position within $1 \mathrm{kB}$ of the peak. (B) Histogram of the top 10,000 peaks in comparison with the distance from the transcriptional start site (TSS), indicating enormous enrichment very close to the TSS. (C) Binding within $\pm 1 \mathrm{kB}$ of the transcriptional start site in each of the conditions tested. Only the control siRNA + DEX condition showed any appreciable binding, as expected. Two small clusters of genes (clusters 1 and 3) had well-defined binding areas with regard to the TSS. The vast majority (cluster 2) had no discernible pattern. (D) Pie chart of the top 10,000 peaks indicating the location of binding in the genome. (E) Characterization of the top 1000 ChIP-seq peak binding sites by location. (F) Sixty-five of 1000 peaks were found to have both the classic GRE motif (top) and a de novo motif (bottom). (C) Binding site by location of the 65 peaks with both motifs.

within $\pm 5 \mathrm{~kb}$ of the ChIP-seq binding site, the majority had either none or only 1 (Figure 2C). This list of 29 genes was again queried by using the ENCODE ChIP-seq Significance Tool and the mm10 reference genome for TF within \pm 500 bp of the TSS/5' end. The results, shown in Supplemental Table 6, are very similar to those of the 65 genes with widely dispersed ChIP-seq binding peaks, suggesting that EC-GR binding is likely influenced by spatially distant regulatory elements.

Characterization of gene expression changes by RNA-seq. To further understand if, and how, the GR binding patterns discovered by ChIP-seq influenced gene expression, RNA-seq was performed using the same experimental groups as for the ChIP-seq analysis in MLECs, except cells were treated with DEX for 18 hours to allow adequate time for transcriptional responses. As a result of the fact that 4 groups were being compared (control siRNA, control siRNA + DEX, GR siRNA, and GR siRNA + DEX), there were over 143,000 independent fold-change calculations. These data were further restricted to those comparisons that had both a significant $P$ value and a significant $Q$ value, resulting in a more manageable list of 902 comparisons. From this group, 231 genes were DEX responsive and 203 genes were differentially regulated by GR. Of the genes regulated by GR, 111 genes were downregulated in the absence of GR (i.e., induced by GR at baseline) (Supplemental Table 7) and 92 genes were upregulated in the absence of GR (i.e., repressed by GR at baseline) (Supplemental Table 8).

Comparison of GR ChIP-seq in ECs s to GR ChIP-seq in A549 cancer cells. To investigate which pathways were most enriched in our data set, Gene Ontology (GO) was used to analyze the top 1000 peaks from our data and those available in ENCODE from a GR ChIP-seq experiment performed in A549 cells (10). Supplemental Figure 1 represents the pie charts from both data sets, showing proportionally similar enrichment in 4 main pathways of interest: (a) Wnt signaling, (b) inflammation by chemokine/cytokine, (c) cadherin signaling, and (d) angiogenesis, suggesting a similar pattern of GR-responsive genes in these 2 cell types.

Independent in vitro and in vivo validation of selected genes identified by GR ChIP-seq. Given that the FDR ( $Q$ value) may be $>0.05$ when dealing with such a large data set, we pursued primer-specific validation of gene targets. Using several genes from each of the 4 pathways of interest, a custom quantitative PCR (qPCR) plate was generated to independently assess expression of these genes in MLECs. Cells were treated with either control or GR siRNA, and levels of gene expression were assessed by qPCR. As shown in Figure 3A, 4 of the 5 genes selected from the Wnt signaling pathway were highly upregulated in the absence of GR. Several genes in the 3 other pathways were also induced by the absence of GR (Supplemental Figure 2, A-C). In our previous study, we demonstrated that ApoE/endothelial GR double KO mice (DKO) developed more severe atherosclerosis and increased inflammation when fed a HFD compared with $A p o e^{-1-}$ mice (7). To determine if these similar pathways were upregulated in vivo, $A p o e^{-/-}$and DKO mice were fed a HFD for 3-4 weeks, and RNA was isolated from the whole aorta for qPCR analysis. As shown in Figure 3B, DKO mice showed increased expression of the selected Wnt genes, consistent with what was observed in vitro. Genes in the inflammation and cadherin pathway also mimicked in vitro results (Supplemental Figure 2, D-F). These results were further verified by using the Integrated Genome Browser program to align the peaks generated by the input DNA and the control siRNA + DEX condition with the reference genome at the chromosomal location indicated by the sequencing data. Alignment data for 3 of these 5 genes are presented in Figure 3, C-E, demonstrating massively enhanced binding when GR is activated by its ligand DEX with very little binding in the input condition.

GR-mediated modulation of the Wnt signaling pathway in vitro. Given the robust effects we observed in the Wnt signaling pathway, as well as the understudied role of Wnt in vascular disease, we focused subsequent efforts on the Wnt pathway. Supplemental Table 9 shows the identity of the genes in the Wnt signaling pathway with GR binding sites with close proximity to the TSS based on intronic peaks.

To directly test how the presence or absence of GR could affect downstream targets of the canonical Wnt signaling pathway, MLECs were treated with Wnt3a conditioned media, in the absence or presence 
A

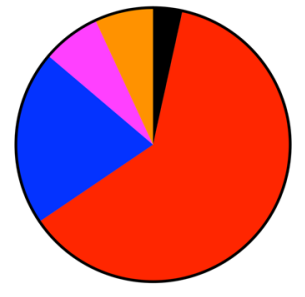

Total $=29$

B

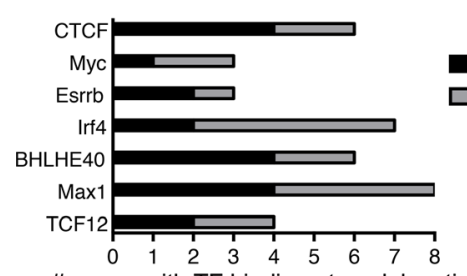

\# genes with TF binding at peak location

\section{C}

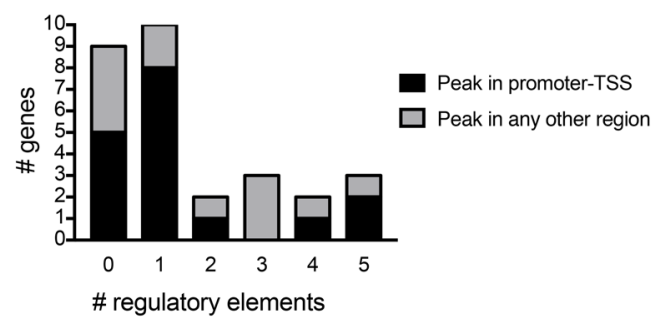

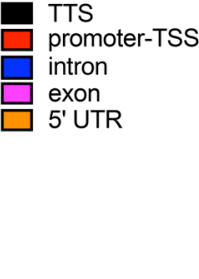

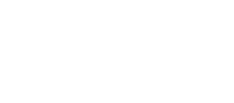

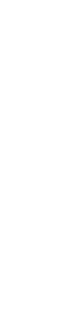

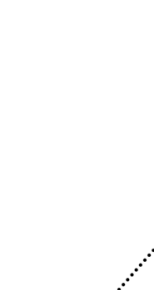

CTCF

Myc
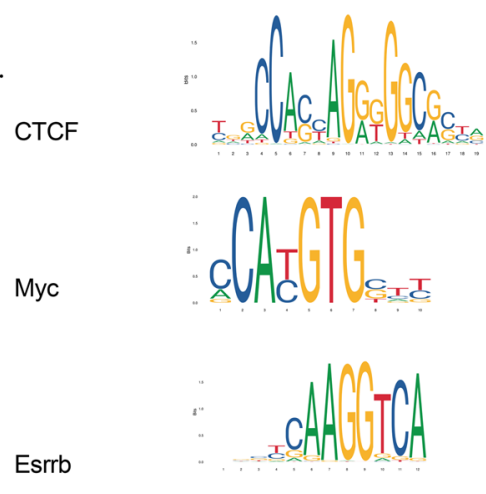

Irf4

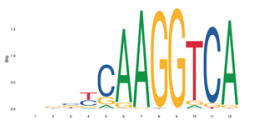

BHLHE40

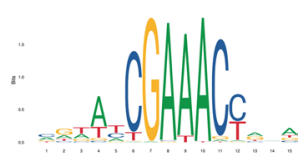

Max1
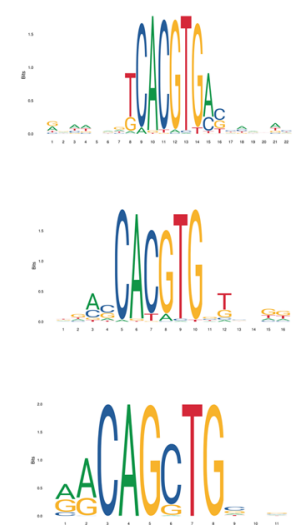

Figure 2. Characterization of $\mathbf{2 9}$ genes identified from the top $\mathbf{1 0 0 0}$ peaks that exhibited ChIP peak binding within $\pm \mathbf{1}$ kb of the transcriptional start site (TSS). (A) ChIP peak binding location by region. (B) The peak location of each of the 29 genes was examined individually in the ENCODE database. The number of genes with transcription factor (TF) binding at the peak location based on the location of the peak binding site is shown. The 7 most abundant transcription factors are quantified, and the corresponding motifs are shown. (C) The number of regulatory elements is quantified as a function of peak location for each of the 29 genes surveyed.

of DEX; then, the expression of sox 17 and axin2, both canonical Wnt-dependent genes, was assessed. As shown in Figure 4A, sox17 expression was induced by Wnt3a, and administration of DEX suppressed expression after 6 hours; a similar pattern was observed for axin2 (Figure 4B). To further examine the specificity of GR in this interaction, a similar experiment in GR siRNA-treated cells was performed. As shown in Figure 4C, GR knockdown caused increased basal sox17 expression in the unstimulated cells, and this enhanced expression was augmented by treatment with Wnt3a. Moreover, DEX treatment suppressed sox17 expression to a much greater extent in control siRNA-treated cells than in GR siRNA-treated cells, demonstrating that GR represses the actions of Wnt3a on sox 17 levels. These results were further verified by developing an EC line with stable expression of a TCF/LEF reporter construct, allowing assessment of canonical activation of the Wnt signaling pathway by quantification of luciferase activity. As shown in Figure 4D, GR knockdown increased luciferase activity, both in the absence and in the presence of the canonical ligand Wnt3a. Protein expression of $\beta$-catenin was significantly increased in vitro with GR knockdown in MLECs (Figure 4E and quantified in Figure 4F).

To test whether Wnt ligands were themselves proinflammatory in our cell culture system, we assessed the expression of the inflammatory markers IL- 6 and TNF- $\alpha$ after treatment with Wnt3a or Wnt5a in control- and GR siRNA-treated MLECs. As shown in Supplemental Figure 3, there was no difference in the expression of IL- 6 mRNA with either Wnt3a or Wnt5a treatment; the expression of TNF- $\alpha$ was slightly decreased in the presence of Wnt5a. Overall, these data suggest that Wnt ligands are not proinflammatory in our model. 
A

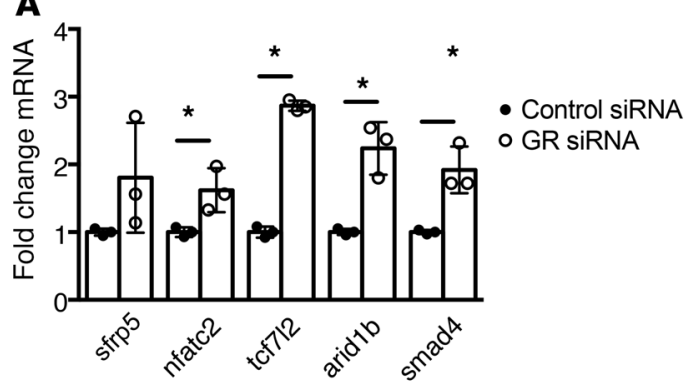

B

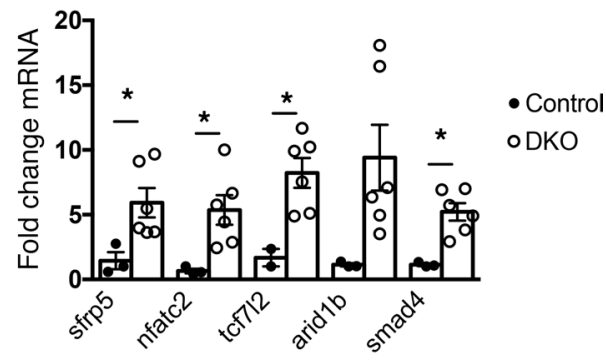

Figure 3. Primer-specific qPCR for genes in the Wnt signaling pathway, which were identified in the top 1000 peaks of the glucocorticoid receptor (GR) ChIP-seq data set, was performed both in vitro and in vivo. (A) Control siRNA- or GR siRNA-treated MLECs. Data represent 3 separate experiments. (B) Double knockout (DKO) $(n=6)$ or control $\left(\right.$ Apoe $\left.^{-/-}\right)$mice $(n=3)$ fed with high-fat diet for 3 weeks. Whole aortas were dissected for RNA isolation and qPCR. Unpaired $t$ test was used for each primer set. ${ }^{*} P<$ 0.05. (C-E) Alignment of the input DNA with the Con DEX condition from the ChIP-seq data demonstrates massively enriched GR binding at the peak locations for Tcf7l2 (C), Arid1b (D), and Smad4 (E).

D
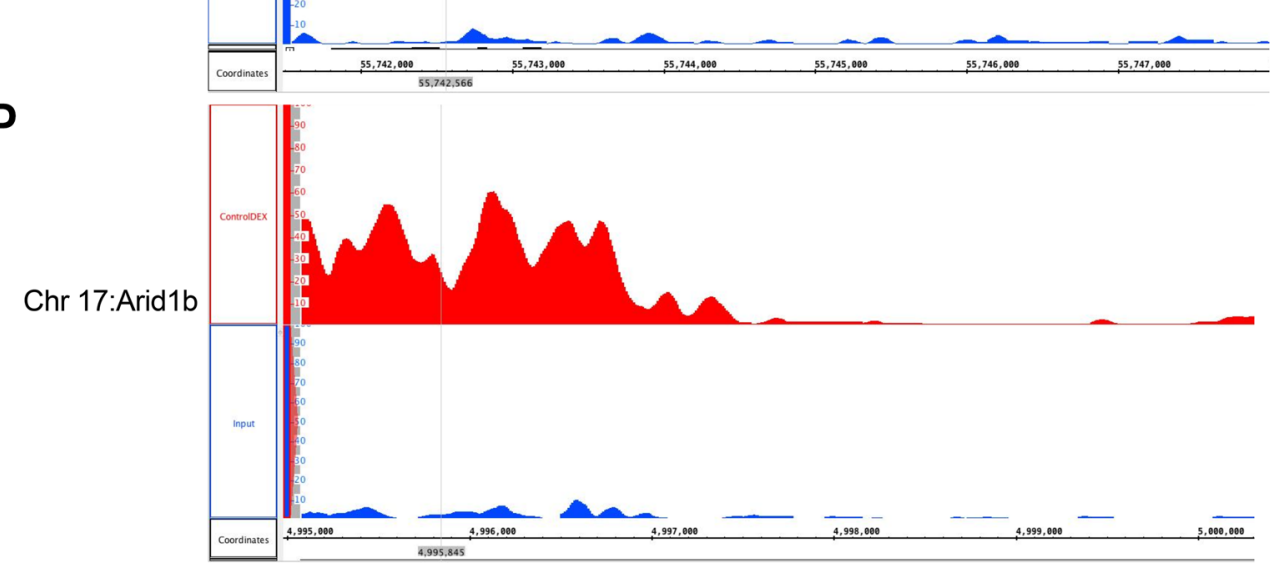

E

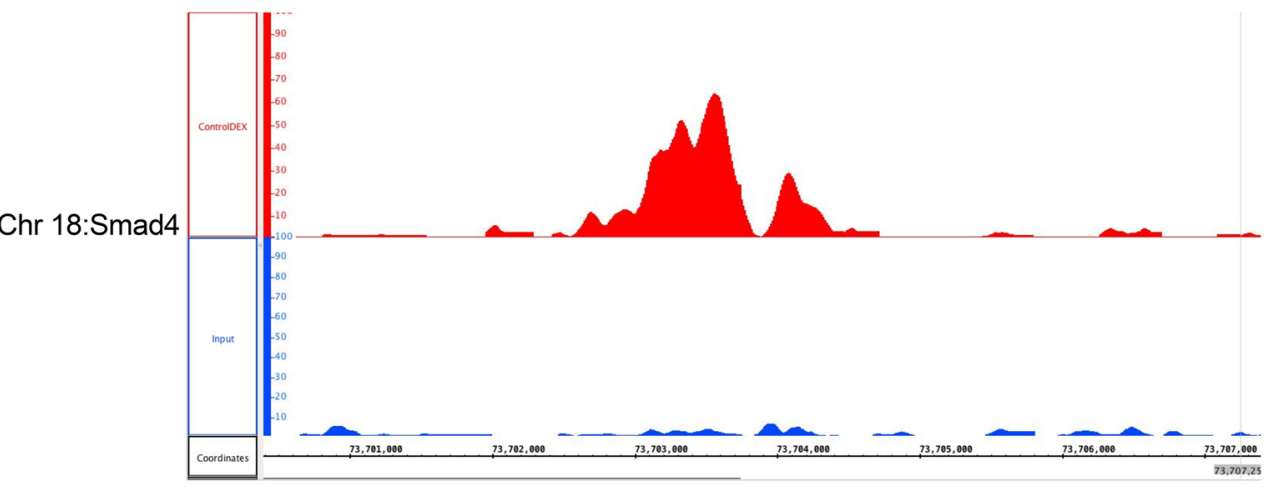

Finally, to verify that the observed responses were mediated via the canonical Wnt signaling pathway, ECs were treated with either control siRNA or with low-density lipoporotein-related receptor 5 (LRP5) siRNA or low-density LRP6 siRNA, 2 key molecules in the initiation of the canonical Wnt signaling pathway. With effective knockdown of either of these receptors, the expression of the Wnt-dependent genes axin2 and sox17 could not be augmented with DEX treatment, confirming the involvement of canonical Wnt signaling (Supplemental Figure 4).

Detection of a novel GRE. A subset of genes in each of the 4 pathways - which were identified in Figure 4 and (a) had peaks in the ChIP-seq data set, (b) were present in clusters 1-3 (as shown in Figure 1C), and (c) had statistically significant fold changes in the RNA levels as determined by both $P$ 
A

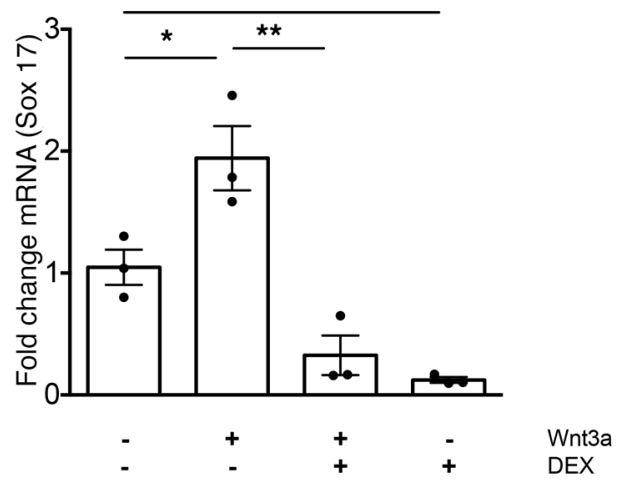

C

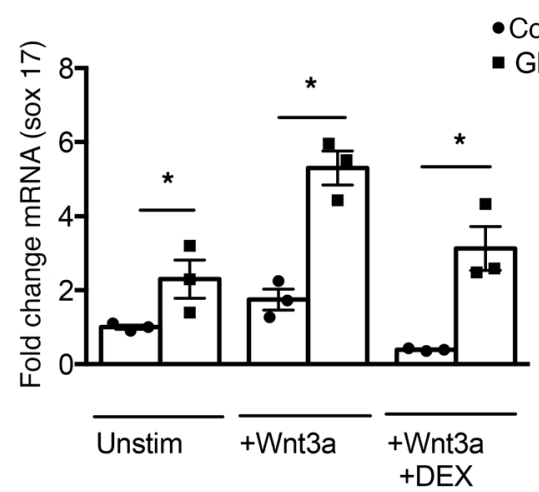

- GR siRNA
B

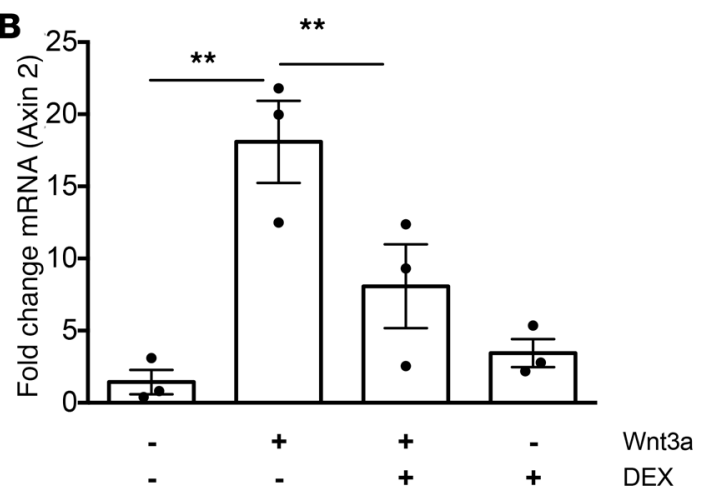

D

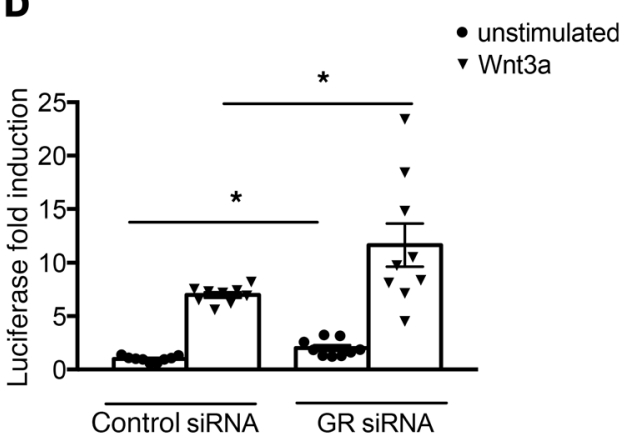

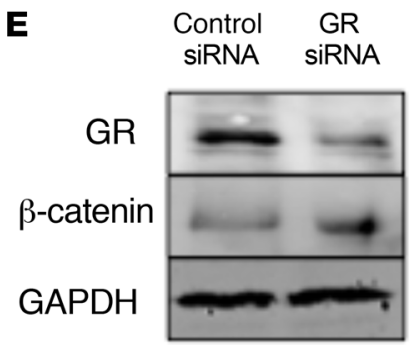

Control siRna

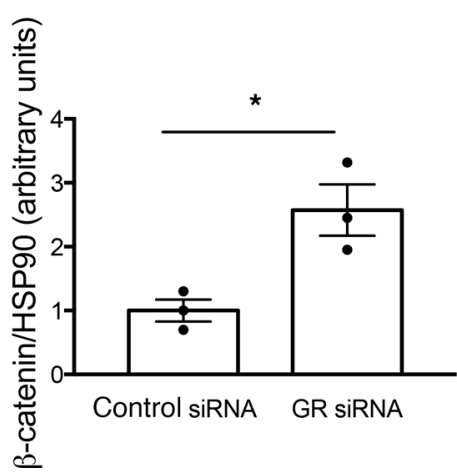

Figure 4. In vitro regulation of canonical Wnt signaling by the glucocorticoid receptor (GR). Mouse lung endothelial cells (MLECs) were serum starved in $0.5 \%$ FBS for 4 hours and then treated with 10\% Wnt3a conditioned media for 6 hours. Dexamethasone (DEX) $100 \mathrm{nM}$ was added for 1 hour at the completion of the media incubation period. (A and B) qPCR for Sox17 (A) and Axin2 (B) was performed. (C) Control siRNA or GR siRNA MLECs were treated with Wnt3a $200 \mathrm{ng} / \mathrm{mL}$ for 4 hours in the presence or absence of DEX $100 \mathrm{nM}$ for 1 hour, and qPCR for Sox 17 was performed. (D) MLECs were stably transfected with a TCF/LEF luciferase construct and subjected to either control siRNA or GR siRNA treatment, with and without Wnt3a $200 \mathrm{mg} / \mathrm{mL}$ for 4 hours. (E) MLECs were treated with either control siRNA or GR siRNA, and lysates were subjected to Western blot for GR and $\beta$-catenin expression. (F) Quantification of GR and $\beta$-catenin expression by densitometry. Data represent 3 independent experiments. One-way ANOVA with Tukey's post hoc test was used to analyze data in $\mathbf{A}, \mathbf{B}$, and $\mathbf{D}$. Student's $t$ test was used to analyze data in $\mathbf{C}$ and $\mathbf{F}$. ${ }^{*} P<0.05 ;{ }^{*} P<0.01$.

and $Q$ values - was interrogated. This resulted in the 16 genes shown in Figure 5A, which are presented as a heatmap analysis of biological replicates from the RNA-seq data. Using motif detection software, this group of genes was queried for conserved motifs, and a potentially new motif was detected as shown in Figure 5B. Previous queries of larger gene subsets had failed to reveal any plausible motif. The genomic sequence of each of these 16 genes was reviewed individually to determine whether the detected motif was present. A perfect match for the motif with guanine residues at positions 15 and 16 was found in intron 5 of the frzb gene at position 80,415,049-80,415,068 on chromosome 2 (entire genomic DNA shown in Supplemental Figure 5). Frzb, a secreted Wnt antagonist, was found to be significantly downregulated in the absence of GR, again supporting the notion that absence of endothelial GR results in heightened Wnt signaling. 


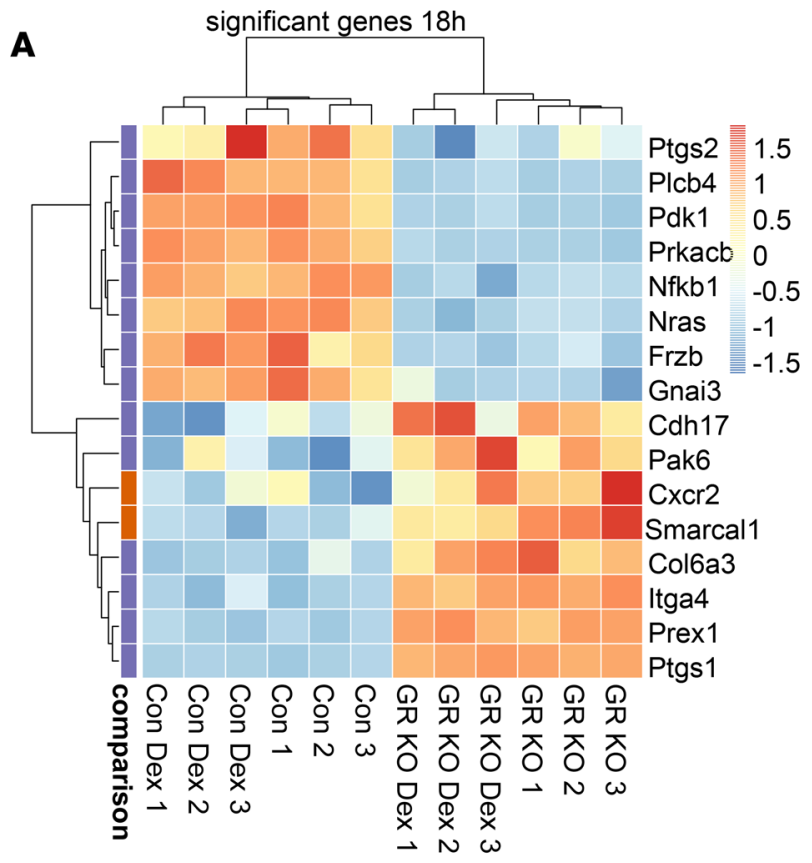

Figure 5. Detection of a potentially novel motif. (A) Heatmap of fold-expression changes of 16 genes identified through advanced analysis of sequencing data. Trends are conserved for both GR replete and GR-KO conditions. (B) Potentially novel motif detected from analysis of 16 genes in A. (C) Luciferase assay showing fold induction for cells treated with media alone, RU486 alone for 4 hours, DEX alone for 4 hours, or RU486 4-hour pretreatment followed by 4 hours with DEX. Data represent 3 separate experiments. One-way ANOVA with Tukey's post hoc test was used to analyze data. ${ }^{*} P<0.05$.

B
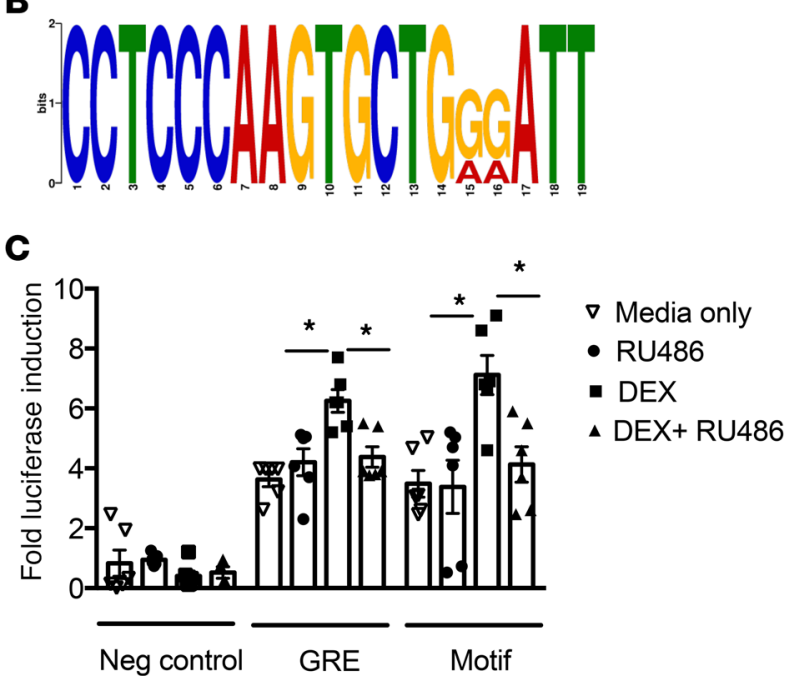

Examination of this region using Ensembl demonstrates that there are several CCCTC-binding factor (CTCF) sites in close proximity to the binding peak. We confirmed frzb-GR binding was indirect by designing 2 different sets of primers for frzb and performing DNA qPCR using the ChIP DNA; frzb was not detected using either primer set. Interestingly, using ChIP input DNA, the loss of GR reduced CTCF expression by more than $80 \%$, suggesting that CTCF may regulate frzb in a spatiotemporal manner via GR as has previously been demonstrated $(21,22)$. We also examined what happened to frzb expression when CTCF was downregulated. As shown in Supplemental Figure 6, A and B, knockdown of CTCF using an siRNA approach resulted in a significant decrease in frzb mRNA expression, further supporting this hypothesis. To investigate the effect of frzb in our model, control and GR siRNA-knockdown ECs were treated with exogenous Sfrp3, and the effect of Wnt-dependent gene expression was assessed. Sfrp3 was able to decrease the expression of axin2 in both control and GR knockdown cells, but this trend result was not observed for sox17. As expected, the expression of both genes was statistically higher in GR knockdown cells (Supplemental Figure 6, C and D).

To test whether this motif demonstrated functional regulation by GR in vitro, several luciferase constructs were generated and expressed into 293T cells. The pGL3 basic vector was used as a negative control, and a previously described GRE (ref. 23 and Supplemental Figure 7A) was used as a 
positive control. The experimental motif was cloned as 4 tandem repeats (Supplemental Figure 7B), while the positive control contained 2 classic consensus GRE sequences derived from the tyrosine aminotransferase (TAT) gene promoter.

As shown in Figure 5C, after 4 hours of treatment with DEX $100 \mathrm{nM}$, the potentially new experimental motif resulted in luciferase activation that was very similar to that observed in the positive control. As expected, the negative control exhibited a very low signal. The activity of both responsive constructs could be significantly downregulated, though not extinguished, by 4 hours of treatment with $1 \mu \mathrm{M}$ RU486, a GR antagonist, and this effect was unable to be reversed with subsequent DEX treatment, validating the receptor-specific response. The high baseline activation observed in the media-only condition - for both the known GRE and the motif, but not the negative control - is a result of small amounts of endogenous steroid in the culture media and further confirms the validity of our assay.

In vivo activation of Wnt signaling in DKO mice. To enable direct visualization of the activation of canonical Wnt signaling in vivo, Apoe $e^{-/}$and DKO mice were bred to the TCF/Lef-LacZ reporter mouse and subjected to 12 weeks of HFD feeding. At the conclusion of the feeding period, aortas were isolated and stained with Oil Red $\mathrm{O}$ and X-galactosidase (X-gal). As shown in representative aortas in Figure 6A and quantified in Figure 6B, DKO animals had significantly more severe atherosclerotic lesions, consistent with previous results (7). LacZ staining in both genotypes, shown in Figure 6C and quantified in Figure 6D, revealed significantly more staining DKO animals, consistent with upregulated canonical Wnt signaling. Aortic ECs isolated from animals of both genotypes at the end of the feeding period showed significantly increased mRNA expression of the Wnt-dependent genes axin2, ctnnb1, and TCF/ Lef(Figure 6E). There were no differences in total cholesterol or triglyceride levels either before or after HFD feeding, consistent with previous results (Supplemental Figure 8).

\section{Discussion}

The major finding of this study is that endothelial GR is an important modulator of the Wnt signaling pathway, which influences inflammation, both in vitro and in vivo. Notably, the repressive effect of GR on the Wnt pathway is independent of the NF- $\mathrm{BB}$ pathway, a classic target for GR, and highlights the permissive role of this receptor in physiologically relevant states.

The Wnt signaling pathway is gaining prominence as an underappreciated player during inflammatory disorders such as atherosclerosis. In vitro, Wnt ligands have been shown to induce EC proliferation and modulate inflammation (24-26). Noncanonical Wnt5a/ $\mathrm{Ca}^{2+}-$ dependent signaling induces endothelial inflammation and release of inflammatory cytokines (27). In vivo, Wnt5a staining is upregulated in both murine and human atherosclerotic plaques (28). Interestingly, nuclear localization of $\beta$-catenin, in the canonical pathway, has been described in athero-prone areas of the aortic endothelium prior to lesion development (29). Sclerostin, an inhibitor of the Wnt pathway, has been found to inhibit atherosclerosis (30); most recently, Wnt5a was shown to mediate lipid accumulation in macrophages and the formation of foam cells in studies of human carotid vessels (31). Furthermore, the Wnt5a-JNK signaling axis has been shown to contribute to the endothelial dysfunction present in human diabetes (32).

There is also evidence from the heart failure literature that the Wnt signaling pathway and its modulators may be important risk factors in human cardiovascular disease. For example, Ueland and colleagues showed that the Wnt modulators secreted frizzled related protein-3 (Sfrp3), dickkopf-1 (DKK-1), and Wnt inhibitory factor 1 (WIF-1) - all of which are Wnt signaling inhibitors — were substantially upregulated in patients with symptomatic severe aortic stenosis (33). The same authors went on to show an association between elevated levels of Sfrp3 and poor outcome in human heart failure patients, as well as elevated Sfrp3 mRNA expression in the left ventricles of postmyocardial infarction mice (34). Interestingly, this study suggested that the increased Sfrp3 was derived, at least in part, from the myocardium itself. The impact of Sfrp3 levels on cardiovascular outcomes was also assessed in patients with chronic heart failure. When Sfrp3 levels were stratified by tertile and examined as a risk factor for any cardiovascular event, somewhat surprisingly, those patients with levels in the middle tertile fared better than those with either very low or very high Sfrp3 levels (35). These data suggest that there may be a "sweet spot" with regard to Wnt activity and cardiovascular health.

These clinical findings are also supported by in vitro data, both from human cells and animal models, that suggest Wnt/ $\beta$-catenin signaling may be both pro- or antiinflammatory based on tissue conditions. Some studies support a negative regulatory role of $\mathrm{NF}-\mathrm{kB}$ signaling by the Wnt pathway and an overall antiinflammatory 
A

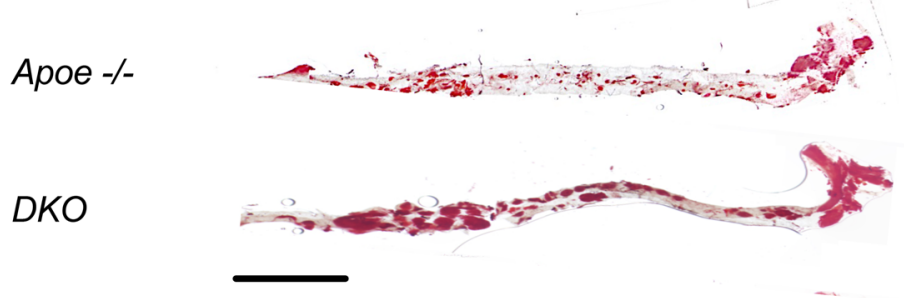

C

Apoe -/LacZ(-)

Apoe -/-
$\operatorname{LacZ}(+)$

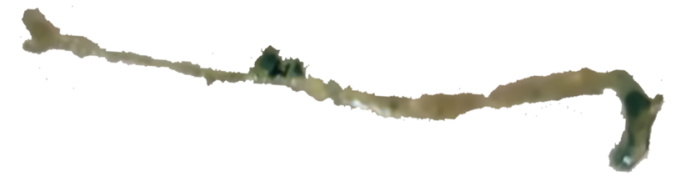

\section{DKO \\ $\operatorname{LacZ}(+)$}

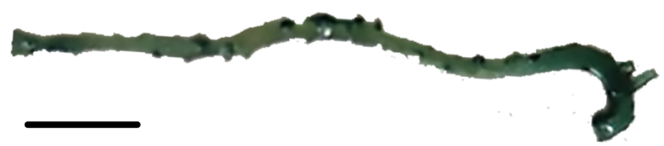

E

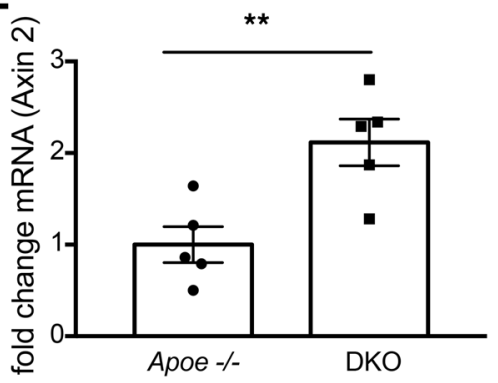

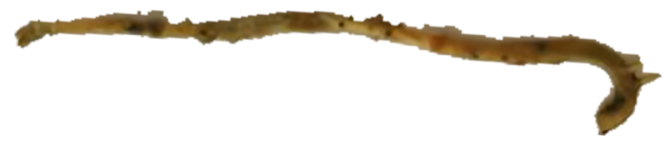

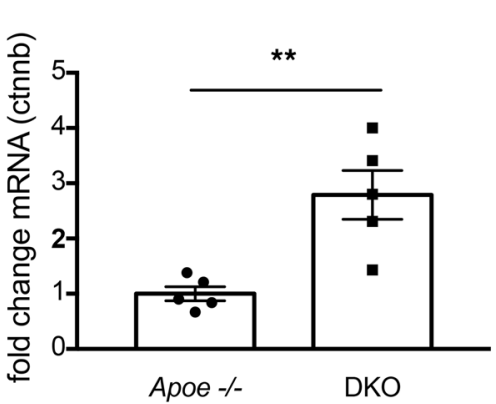

B
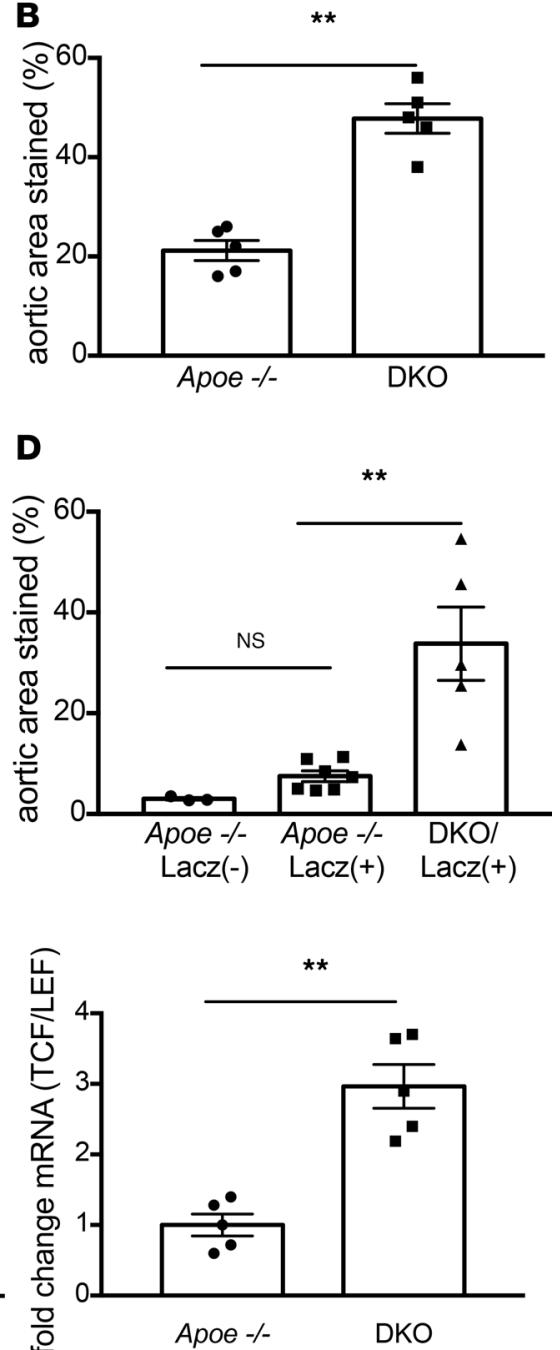

Figure 6. In vivo regulation of canonical Wnt signaling. (A) Representative aortas stained with Oil Red O from Apoe ${ }^{-/-}$and double KO (DKO) mice. Scale bar: $1 \mathrm{~cm}$. (B) Quantification of lesion size in each genotype ( $n=5 /$ group). (C) Representative aortas stained with X-gal demonstrate significantly higher $\beta$-galactosidase staining in DKO mice. A LacZ(-) control is included for comparison. Scale bar: $1 \mathrm{~cm}$. (D) Quantification of $\beta$-gal expression in each genotype ( $n$ = 3-7 mice/group). (E) mRNA expression of Axin 2, ctnnb, and TCF/LEF is significantly higher in aortic endothelial cells from DKO mice than Apoe ${ }^{-/-}$mice after diet feeding ( $n=5$ /group). Student's $t$ test was used to analyze data. ${ }^{* *} P<0.05$.

effect of Wnt signaling, though there are also some that have reported positive stimulation of NF-kB signaling by Wnt (36). For example, Wnt5a has been shown to activate RelA nuclear translocation and DNA binding, and activated blood vessels in both atherosclerosis and rheumatoid arthritis conditions express Wnt5a, suggesting a proinflammatory role for this ligand in vivo (27). Further evidence for this phenomenon was shown by Yang et al., who demonstrated inhibition of both NF-kB signaling and MAPK signaling after in vivo silencing of Wnt5a in a mouse model of atherosclerosis (37). Wnt5a - as well as other proteins central to the Wnt and NF-kB pathways, including JNK1 and NF- $\mathrm{kB}$ p 65 - have been shown to be significantly upregulated in an animal model of hepatic inflammation induced by type 2 diabetes mellitus and nonalcoholic steatohepatitis (38); interestingly, Wnt5a reduction is regarded as a potential strategy for macrophage differentiation and enhanced cell apoptosis in Mycobacterium tuberculosis-infected mice (39) There is also a large body of literature that provides strong evidence for a negative regulatory role of Wnt signaling on NF-kB signaling, much of which has been derived from the study of cancer cells. In particular, studies in colon cancer and breast cancer cells subjected to overexpression of $\beta$-catenin have shown that the NF-kB signaling pathway is inhibited due to the formation of a $\beta$-catenin-RelA-p50 complex, which decreases NF- $\mathrm{kB}$ transactivation and proinflammatory gene transcription (40-42). Similar to the clinical experience, these preclinical and basic studies reveal a dichotomous role of Wnt signaling, which seems to depend on both specific Wnt ligands and cell types. 
In this study, we show conclusively, using both next-generation sequencing techniques and a potentially novel mouse model, that endothelial GR is an important regulator of the canonical Wnt signaling pathway. Our results are in good agreement with ChIP-seq experiments done in A549 cells, which also show proportionately increased binding of Wnt-related genes based on publicly available data in the ENCODE database (https://www.encodeproject.org). Independent validation of selected targets in vitro and in vivo using our published DKO mouse model further validated these results.

One limitation of these data is that the analysis was restricted to peaks in close proximity to TSS and the corresponding RNA-seq data. However, the mechanisms of GR effects on cellular responses, transcriptional or otherwise, remain difficult to classify by any straightforward categorization scheme. For example, Vockley et al. have recently shown in human epithelial cells that direct GR binding sites may interact with nonresponsive sites via enhancers or other epigenetic modifications over many tens of kilobases (13). Jubb et. al. demonstrated that large-scale chromatin decompaction in macrophages, which was not dependent on transcription, occurred rapidly upon GR binding and could influence glucocorticoid response over many hours, even when ligand was removed (43). Similarly, in experiments examining GR-mediated repression of proinflammatory genes in mouse macrophages, at least 2 discrete mechanisms were present: (a) promoter accumulation of negative elongation factor (NELF) and (b) blocking of RNA polymerase (Pol) 2 activation (44). It should be noted that these mechanisms, demonstrated in epithelial cells and macrophages, may not be generalizable to ECs, given the exquisite control of GR-mediated responses in specific cell types, a phenomenon that is increasingly recognized. There is much that remains to be learned about the detailed mechanisms of GR effects on cellular responses.

Another limitation of the ChIP-seq approach is that the peak calling software uses the nearest gene to the peak as the predicted binding site, which may not always be accurate. Indeed, when the peak for frzb was reviewed, we noted that the software designated the binding site as intergenic, which would not explain the intronic motif that was detected. It remains possible that there is an intergenic binding site close to $f r z b$ that was not uncovered in the subsequent analyses, though this seems somewhat unlikely given the excellent luciferase induction that was achieved with DEX treatment. A further uncertainty of the ChIPseq approach is that binding cannot be determined as direct or indirect with this methodology. The ChIP DNA qPCR and data from CTCF siRNA knockdown support the notion that frzb-GR binding is indirect and potentially mediated via CTCF. Further study will be required to investigate this possibility. While GR binding was clearly enriched in the DEX-treated control group, as expected, it is not known how much of this binding was mediated directly through GR versus any other intermediary genes.

Integration of the ChIP-seq and RNA-seq data sets revealed that even the most differentially regulated GR-dependent genes did not necessarily have peaks in the ChIP-seq data set and, if present, were not among the most highly enriched peaks. Of course, there may be other genes in the RNA-seq data set that are significantly regulated by GR and were not considered in this analysis since genes were restricted to those with both significant $P$ and $Q$ values. Detection of these genes on a larger scale would require individual primer design based on the binding site resulted from the peak calling software and qPCR for each target of interest.

In conclusion, we present an endothelial GR-Wnt interaction that may explain the striking phenotypes of heightened atherosclerosis in DKO mice (7) or impaired survival after low-dose LPS in EC-specific GR-KO mice (6), 2 phenotypes largely dependent on endothelial GR. Inhibition of the Wnt signaling pathway in ECs may prove to be a valuable therapeutic opportunity for inflammatory disorders. Ongoing study of the ways in which cell-specific GR directs target gene transcription continues to reveal an increasingly complex regulatory system.

\section{Methods}

Reagents. DEX phosphate was purchased from MP Biomedicals. Recombinant mouse Wnt3a protein was purchased from Abcam. AllStars negative control siRNA and Stealth RNAi for NR3C1 were from Qiagen. Recombinant mouse sFRP3 protein was purchased from Thermo Fisher Scientific. CTCF siRNA was from Dharmacon, and LRP5 and LRP6 siRNA were from Santa Cruz Biotechnology Inc.

ChIP-seq. ChIP-seq was performed using the EZ-ChIP kit (MilliporeSigma) according to the manufacturer's instructions. A total of $5 \mu \mathrm{g}$ of ChIP-grade GR antibody (Abcam, 3578) was used for each experimental condition. GR-enriched ChIP DNA was confirmed by qPCR using primers for Per1. Data analysis was provided by the Yale Center for Genomic Analysis as follows: The fastq files obtained from the sequencer were trimmed for quality using fastx_trimmer. The reads were aligned 
to the mm10 (University of California Santa Cruz [UCSC], Santa Cruz, California, USA) version of the mouse genome using bwa mem. Peaks were called using macs peak-caller (45), and the reads from input DNA sample were used as control. Visualization of the peaks was done using R (cummeRbund), and Integrated Genome Browser using .bw files was used to generate visual images of the experimental conditions with the reference sequence. These data have been assigned GEO accession number GSE119093.

Custom $q$ PCR. A custom PCR array was designed with the Qiagen on-line tool (Custom RT ${ }^{2}$ Profiler PCR Arrays). MLECs were treated with control or GR siRNA for 48 hours. Total RNA was isolated using the RNeasy kit (Qiagen), and cDNA was generated using the iScript cDNA synthesis kit (Bio-Rad) using $1 \mu \mathrm{g}$ RNA. The PCR array was run using SYBR green reagents according to the manufacturer's instructions.

Primer-specific qPCR. ChIP DNA or total RNA was used for qPCR. Total RNA was isolated using standard Trizol protocol. RNA was reverse transcribed using the iScript cDNA Synthesis kit (Bio-Rad), and qPCR was performed on a Bio-Rad C1000 Touch thermal cycler using the resultant cDNA, qPCR master mix, and gene-specific primers. The following primers were used: Per1, forward, 5' - AAGGCTGTGTGCATGTCCT - 3', and reverse, 5' - AGAGGGAGGTGACGTCAAAG - 3'; Sox17, forward, 5' - TCAGATGTCTGGAGGTGCTG - 3', and reverse, 5' - TGGAACCTCCAGTAAGCCAG 3'; Axin2, forward, 5' - AACCTATGCCCGTTTCCTCTA - 3', and reverse, 5' - GAGTGTAAAGACTTGGTCCACC - 3'; Wnt3a, forward, 5' - CTCCTCTCGGATACCTCTTAGTG - 3', and reverse 5' - CCAAGGACCACCAGATCGG - 3'; Ctnnb1, forward, 5' - TGACACCTCCCAAGTCCTTT - 3', and reverse, 5' - TTGCATACTGCCCGTCAAT - 3'; TCF, forward, 5' - GGTGGCCGAATGCACATTGAAAGA - 3', and reverse, 5' - TTTGCCTGTTCTTCCCTGGACA - 3'; Frzb primer 1, forward, 5' - TCTCAATGTATCACTCTGTG - 3', and reverse, 5' - TTTCCAAGAGTTGTTTGTG - 3'; Frzb primer 2, forward, 5' - TCTGTCCCCAAAGAGACATAT - 3', and reverse, 5' - TGGCCTACTATTAAGAGAAA - 3'; CTCF, forward, 5' - CATCATTCAACGTTTAGTTT - 3', and reverse, 5' - GCCAGGGCTATACAGAGAAAC - 3'; IL-6, forward, 5' - TCTGAAGGACTCTGGCTTTG - 3', and reverse, 5' - GATGGATGCTACCAAACTGGA - 3'; and TNF- $\alpha$, forward, 5' - AGGGTCTGGGCCATAGAACT - 3', and reverse, 5' - CCACCACGCTCTTCTGTCTAC - 3'.

Gene expression was normalized to the housekeeping gene $18 \mathrm{~s}$ and is presented as fold change.

Stable cell line. The Cignal Lenti TCF/LEF Reporter (Qiagen) was transduced into MLECs using SureENTRY Transduction Reagent (Qiagen) according to the manufacturer's instructions.

Animal studies. Male Apoe $e^{-/}$and endothelial GR/ApoE-double KO (DKO) mice, 10-12 weeks of age, were previously generated in the lab and fed a HFD containing $1.25 \%$ cholesterol (Research Diets) for the time indicated. In some experiments, animals of both genotype were bred to the BAT-GAL ( $\beta$-catenin/TCF-LEF) reporter transgenic mouse. Mice were sacrificed, and the aortas were removed. Aortas were stained with X-gal (Sigma-Aldrich) according to the manufacturer's instructions. In other aortas, total RNA was extracted and cDNA was made as described above. The custom PCR array was used to evaluate pathways of interest as described.

RNA-seq. MLECs were cultured in EBM-2 and 10\% serum. Cells were treated with control or GR siRNA for 48 hours with and without DEX 100 nM for 18 hours. Total RNA was isolated using the RNeasy kit (Qiagen) and sequenced on an Illumina platform by the Yale Center for Genomic Analysis. Data analysis was provided by the Yale Center for Genomic Analysis as follows: The fastq files obtained from the sequencer were trimmed for quality using fastx_trimmer. The reads were then aligned to the mm10 (UCSC) version of the mouse genome using TopHat (46). The transcript abundance estimation and differential gene expression was carried out using cuffdiff (cufflinks) (47). The results were visualized using R (cummeRbund). These data have been assigned GEO accession number GSE119093.

Motif detection. MEME was used for de novo motif detection, and MAST (48) was used to scan the sequences for known motifs.

Cell culture studies. MLECS were cultured and starved for 4 hours in $0.5 \%$ FBS. Cells were treated for 4 hours with Wnt3a (200 ng/mL). At the conclusion of the 4-hour time period, cells were additionally treated with $100 \mathrm{nM}$ DEX for 1 hour. In other experiments, cells were treated with $10 \% \mathrm{Wnt} 3$ a conditioned media for 6 hours, followed by DEX $100 \mathrm{nM}$ for 1 hour. In other experiments, cells were also treated with Wnt5a (150 ng/mL) and in others cells were treated with recombinant Sfrp3 $(200 \mathrm{ng} / \mathrm{mL})$ for 4 hours.

Plasmid constructs and luciferase assay. The synthetically synthesized gBlocks Gene Fragments (ITD Integrated Technologies) containing $4 \mathrm{GR}$ motifs (underlined) and homologues region to the destination vector (italics), GAACATTTCTCTATCGATAAGGTACCctctgCCTCCCAAGTGCTGGGATTaaagg- 
cgtgactctgCCTCCCAAGTGCTGGGATTaaaggcgtgactctgCCTCCCAAGTGCTGGGATTaaaggcgtgactctgCCTCCCAAGTGCTGGGATTaaaggcgtgaCTCGAGATCTGCGATCTGCATCTCAA, were cloned between KpnI and XhoI into pGL3-Promoter Vector (Promega) using DNA assembly strategy (NEBuilder HiFi DNA Assembly Kit, New England Biolabs). The GR motif is followed by an SV40 promoter and then by the luciferase gene; the quantified luciferase expression is a direct measure of transcriptional activity. The empty pGL3 promoter vector was used as a negative control. 293T cells were transformed with Lipofectamine (Invitrogen).

Plasma measurements. Mice were fasted for 12-15 hours, and blood was collected by retro-orbital venous puncture. Whole blood was spun down, and plasma was stored at $-80^{\circ} \mathrm{C}$. Total cholesterol and triglyceride levels were measured enzymatically by kits from Wako and MilliporeSigma (Wako Diagnostics Total Cholesterol E and Triglyceride Quantification Colorimetric/Fluorometric Kit), respectively, according to the manufacturer's instructions.

Atherosclerotic lesion analysis. At the completion of HFD feeding, mice were anesthetized and euthanized. Mouse hearts were perfused with PBS and then 4\% paraformaldehyde (PFA); the aortas and were dissected out using a dissecting microscope and maintained in PFA overnight. Whole aortas were stained with Oil Red O (MilliporeSigma) to quantify lesion area. Oil Red O stock solution ( $35 \mathrm{~mL}, 0.2 \%$ weight/ volume in methanol) was mixed with $10 \mathrm{~mL} 1 \mathrm{M} \mathrm{NaOH}$ and filtered. Aortas were briefly rinsed in $78 \%$ methanol, incubated in Oil Red O for 45 minutes, and destained in $78 \%$ methanol for 5 minutes, and mounted on microscopic slides. Lipid staining and lesion size were quantified by averaging 6 sections from the same mouse using the ImageJ program (NIH).

Western blot. Tissues were snap frozen in liquid nitrogen, pulverized, and resuspended in lysis buffer (50 $\mathrm{mM}$ Tris $\cdot \mathrm{HCl}$, pH 7.4, 0.1 mM EDTA, 0.1 mM EGTA, 1\% nonidet P-40, 0.1\% sodium deoxycholate, $0.1 \%$ SDS, $100 \mathrm{mM} \mathrm{NaCl}, 10 \mathrm{mM} \mathrm{NaF}, 1 \mathrm{mM}$ sodium pyrophosphate, $1 \mathrm{mM}$ sodium orthovanadate, $1 \mathrm{mM}$ Pefabloc SC, and $2 \mathrm{mg} / \mathrm{mL}$ protease inhibitor mixture; Roche Diagnostics). Cells were lysed on ice with lysis buffer. Protein concentrations were determined with the DC Protein assay kit (Bio-Rad). Lysates were analyzed by SDS/PAGE and immunoblotted. Primary antibodies used include the following: GR (Thermo Fisher Scientific, MA1-510), $\beta$-catenin (BD Biosciences, 610153), and Hsp90 (Affinity Bioreagents, MA3-011). Secondary antibodies were fluorescence-labeled antibodies (LI-COR Biosciences). Bands were visualized with the Odyssey Infrared LI-COR system.

Isolation of primary aortic ECs. Primary cells were isolated as described (49). Briefly, mice were sacrificed, and thoracic aortas were dissected out, opened longitudinally, placed lumen side-down in collagen-coated wells, and covered by culture medium (high glucose DMEM + 20\% FBS). After 2 days in culture, tissue was removed and cells were trypsinized, resuspended, sorted by rat anti-mouse CD31-coated (clone 390, catalog 558736, BD Bioscience) Dynabeads using a magnetic separator, and replated. When subconfluent, they were subjected to second sorting with rat anti-mouse CD102-coated (BD Biosciences) Dynabeads using a magnetic separator and replated for experiments.

Statistics. Binary comparisons were analyzed using Student's 2-tailed $t$ tests. Multiple comparisons were analyzed using 1-way ANOVA with Tukey's post hoc test. Data are expressed as mean \pm SEM. A $P$ value less than 0.05 was considered significant, and where indicated, a $Q$ value of less than 0.05 was also considered significant.

Study approval. All studies were performed according to a protocol approved by the IACUC at Yale University School of Medicine, New Haven, Connecticut, USA, and were consistent with the Guide for the Care and Use of Laboratory Animals (National Academies Press, 2011).

\section{Author contributions}

WCS and JEG conceptualized the study. WCS, CFH, and JEG developed the methodology. SM and JEG validated data. HZ, SM, SPS, and JEG engaged in formal analysis. HZ, SPS, KG, CW, XZ, AH, and PP performed experiments. SM was responsible for resources and data curation. JG wrote the original draft and acquired funding. WS, CFH, and JG supervised the study.

\section{Acknowledgments}

This work was supported by NIH grant R01HL131952 to JG.

Address correspondence to: Julie Goodwin, Yale University School of Medicine, 333 Cedar Street, PO Box 208064, New Haven, Connecticut 06520, USA. Phone: 203.785.4643; Email: julie.goodwin@yale.edu. 
1. Arnett JC, Hatch HB. Diagnostic procedures and clinical management. Chest. 1976;70(6):796-797.

2. Ait-Oufella H, Maury E, Lehoux S, Guidet B, Offenstadt G. The endothelium: physiological functions and role in microcirculatory failure during severe sepsis. Intensive Care Med. 2010;36(8):1286-1298.

3. Lorant DE, Zimmerman GA, McIntyre TM, Prescott SM. Platelet-activating factor mediates procoagulant activity on the surface of endothelial cells by promoting leukocyte adhesion. Semin Cell Biol. 1995;6(5):295-303.

4. Lee JI, Burckart GJ. Nuclear factor kappa B: important transcription factor and therapeutic target. J Clin Pharmacol. 1998;38(11):981-993.

5. Longui CA. Glucocorticoid therapy: minimizing side effects. J Pediatr (Rio J). 2007;83(5 Suppl):S163-S177.

6. Goodwin JE, Feng Y, Velazquez H, Sessa WC. Endothelial glucocorticoid receptor is required for protection against sepsis. Proc Natl Acad Sci USA. 2013;110(1):306-311.

7. Goodwin JE, et al. Endothelial glucocorticoid receptor suppresses atherogenesis--brief report. Arterioscler Thromb Vasc Biol. 2015;35(4):779-782

8. King EM, Holden NS, Gong W, Rider CF, Newton R. Inhibition of NF-kappaB-dependent transcription by MKP-1: transcriptional repression by glucocorticoids occurring via p38 MAPK. J Biol Chem. 2009;284(39):26803-26815.

9. Meduri GU, Muthiah MP, Carratu P, Eltorky M, Chrousos GP. Nuclear factor-kappaB- and glucocorticoid receptor alpha- mediated mechanisms in the regulation of systemic and pulmonary inflammation during sepsis and acute respiratory distress syndrome. Evidence for inflammation-induced target tissue resistance to glucocorticoids. Neuroimmunomodulation. 2005;12(6):321-338.

10. Reddy TE, et al. Genomic determination of the glucocorticoid response reveals unexpected mechanisms of gene regulation. Genome Res. 2009;19(12):2163-2171.

11. Steinhauser S, Kurzawa N, Eils R, Herrmann C. A comprehensive comparison of tools for differential ChIP-seq analysis. Brief Bioinformatics. 2016;17(6):953-966

12. Falkner KC, Ritter JK, Prough RA. Regulation of the rat UGT1A6 by glucocorticoids involves a cryptic glucocorticoid response element. Drug Metab Dispos. 2008;36(2):409-417.

13. Vockley CM, et al. Direct GR Binding Sites Potentiate Clusters of TF Binding across the Human Genome. Cell. 2016;166(5):1269-1281.e19.

14. McEwan MV, Eccles MR, Horsfield JA. Cohesin is required for activation of MYC by estradiol. PLoS ONE. 2012;7(11):e49160.

15. Latos PA, Goncalves A, Oxley D, Mohammed H, Turro E, Hemberger M. Fgf and Esrrb integrate epigenetic and transcriptional networks that regulate self-renewal of trophoblast stem cells. Nat Commun. 2015;6:7776.

16. Carreras E, et al. Estrogen receptor signaling promotes dendritic cell differentiation by increasing expression of the transcription factor IRF4. Blood. 2010;115(2):238-246.

17. Enuka Y, et al. Epigenetic mechanisms underlie the crosstalk between growth factors and a steroid hormone. Nucleic Acids Res. 2017;45(22):12681-12699.

18. Sárvári M, Kalló I, Hrabovszky E, Solymosi N, Rodolosse A, Liposits Z. Long-Term Estrogen Receptor Beta Agonist Treatment Modifies the Hippocampal Transcriptome in Middle-Aged Ovariectomized Rats. Front Cell Neurosci. 2016;10:149.

19. Duffy DJ, et al. Integrative omics reveals MYCN as a global suppressor of cellular signalling and enables network-based therapeutic target discovery in neuroblastoma. Oncotarget. 2015;6(41):43182-43201.

20. Pecenova L, Farkas R. Multiple functions and essential roles of nuclear receptor coactivators of bHLH-PAS family. Endocr Regul. 2016;50(3):165-181.

21. Nakamoto M, et al. The Glucocorticoid Receptor Regulates the ANGPTL4 Gene in a CTCF-Mediated Chromatin Context in Human Hepatic Cells. PLoS ONE. 2017;12(1):e0169225.

22. Starick SR, et al. ChIP-exo signal associated with DNA-binding motifs provides insight into the genomic binding of the glucocorticoid receptor and cooperating transcription factors. Genome Res. 2015;25(6):825-835.

23. Dendoncker K, Timmermans S, Van Looveren K, De Cauwer L, De Bosscher K, Libert C. The nature of the GRE influences the screening for GR-activity enhancing modulators. PLoS ONE. 2017;12(7):e0181101.

24. Goodwin AM, Kitajewski J, D'Amore PA. Wnt1 and Wnt5a affect endothelial proliferation and capillary length; Wnt2 does not. Growth Factors. 2007;25(1):25-32.

25. Masckauchán TN, Shawber CJ, Funahashi Y, Li CM, Kitajewski J. Wnt/beta-catenin signaling induces proliferation, survival and interleukin-8 in human endothelial cells. Angiogenesis. 2005;8(1):43-51.

26. Lee JG, Heur M. WNT10B enhances proliferation through $\beta$-catenin and RAC1 GTPase in human corneal endothelial cells. J Biol Chem. 2015;290(44):26752-26764.

27. Kim J, et al. Wnt5a induces endothelial inflammation via beta-catenin-independent signaling. J Immunol. 2010;185(2):1274-1282.

28. Christman MA, et al. Wnt5a is expressed in murine and human atherosclerotic lesions. Am J Physiol Heart Circ Physiol. 2008;294(6):H2864-H2870.

29. Gelfand BD, et al. Hemodynamic activation of beta-catenin and T-cell-specific transcription factor signaling in vascular endothelium regulates fibronectin expression. Arterioscler Thromb Vasc Biol. 2011;31(7):1625-1633.

30. Krishna SM, et al. Wnt Signaling Pathway Inhibitor Sclerostin Inhibits Angiotensin II-Induced Aortic Aneurysm and Atherosclerosis. Arterioscler Thromb Vasc Biol. 2017;37(3):553-566.

31. Ackers I, Szymanski C, Duckett KJ, Consitt LA, Silver MJ, Malgor R. Blocking Wnt5a signaling decreases CD36 expression and foam cell formation in atherosclerosis. Cardiovasc Pathol. 2018;34:1-8.

32. Bretón-Romero R, et al. Endothelial Dysfunction in Human Diabetes Is Mediated by Wnt5a-JNK Signaling. Arterioscler Thromb Vasc Biol. 2016;36(3):561-569.

33. Askevold ET, et al. Secreted Wnt modulators in symptomatic aortic stenosis. J Am Heart Assoc. 2012;1(6):e002261.

34. Askevold ET, et al. The cardiokine secreted Frizzled-related protein 3, a modulator of Wnt signalling, in clinical and experimental heart failure. J Intern Med. 2014;275(6):621-630.

35. Askevold ET, et al. Secreted Frizzled Related Protein 3 in Chronic Heart Failure: Analysis from the Controlled Rosuvastatin Multinational Trial in Heart Failure (CORONA). PLoS ONE. 2015;10(8):e0133970.

36. Ma B, Hottiger MO. Crosstalk between Wnt/ $\beta$-Catenin and NF-кB Signaling Pathway during Inflammation. Front Immunol. $2016 ; 7: 378$. 
37. Yang L, et al. siRNA-mediated silencing of Wnt5a regulates inflammatory responses in atherosclerosis through the MAPK/ NF-кB pathways. Int J Mol Med. 2014;34(4):1147-1152.

38. Tian F, Zhang YJ, Li Y, Xie Y. Celecoxib ameliorates non-alcoholic steatohepatitis in type 2 diabetic rats via suppression of the non-canonical Wnt signaling pathway expression. PLoS ONE. 2014;9(1):e83819.

39. Chen D, Li G, Fu X, Li P, Zhang J, Luo L. Wnt5a Deficiency Regulates Inflammatory Cytokine Secretion, Polarization, and Apoptosis in Mycobacterium tuberculosis-Infected Macrophages. DNA Cell Biol. 2017;36(1):58-66.

40. Deng J, et al. beta-catenin interacts with and inhibits NF-kappa B in human colon and breast cancer. Cancer Cell. 2002;2(4):323-334.

41. Liu J, et al. PI3K is required for the physical interaction and functional inhibition of NF- $\mathrm{KB}$ by $\beta$-catenin in colorectal cancer cells. Biochem Biophys Res Commun. 2013;434(4):760-766.

42. Du Q, et al. Wnt/beta-catenin signaling regulates cytokine-induced human inducible nitric oxide synthase expression by inhibiting nuclear factor-kappaB activation in cancer cells. Cancer Res. 2009;69(9):3764-3771.

43. Jubb AW, Boyle S, Hume DA, Bickmore WA. Glucocorticoid Receptor Binding Induces Rapid and Prolonged Large-Scale Chromatin Decompaction at Multiple Target Loci. Cell Rep. 2017;21(11):3022-3031.

44. Sacta MA, et al. Gene-specific mechanisms direct glucocorticoid-receptor-driven repression of inflammatory response genes in macrophages. Elife. 2018;7:e34864.

45. Zhang Y, et al. Model-based analysis of ChIP-Seq (MACS). Genome Biol. 2008;9(9):R137.

46. Trapnell C, Pachter L, Salzberg SL. TopHat: discovering splice junctions with RNA-Seq. Bioinformatics. 2009;25(9):1105-1111.

47. Trapnell C, et al. Differential gene and transcript expression analysis of RNA-seq experiments with TopHat and Cufflinks. Nat Protoc. 2012;7(3):562-578.

48. Bailey TL, Bodén M, Whitington T, Machanick P. The value of position-specific priors in motif discovery using MEME. BMC Bioinformatics. 2010;11:179.

49. Molina-Sánchez P, Andrés V. Isolation of Mouse Primary Aortic Endothelial Cells by Selection with Specific Antibodies. Methods Mol Biol. 2015;1339:111-117. 